\title{
Antenatal magnesium individual participant data international collaboration: assessing the benefits for babies using the best level of evidence (AMICABLE)
}

for The AMICABLE Group ${ }^{1}$

\begin{abstract}
Background: The primary aim of this study is to assess, using individual participant data (IPD) meta-analysis, the effects of administration of antenatal magnesium sulphate given to women at risk of preterm birth on important clinical outcomes for their child such as death and neurosensory disability. The secondary aim is to determine whether treatment effects differ depending on important pre-specified participant and treatment characteristics, such as reasons at risk of preterm birth, gestational age, or type, dose and mode of administration of magnesium sulphate.
\end{abstract}

\section{Methods}

Design: The Antenatal Magnesium Individual Participant Data (IPD) International Collaboration: assessing the benefits for babies using the best level of evidence (AMICABLE) Group will perform an IPD meta-analysis to answer these important clinical questions.

Setting/Timeline: The AMICABLE Group was formed in 2009 with data collection commencing late 2010. Inclusion Criteria: Five trials involving a total 6,145 babies are eligible for inclusion in the IPD meta-analysis. Primary study outcomes: For the infants/children: Death or cerebral palsy. For the women: Any severe maternal outcome potentially related to treatment (death, respiratory arrest or cardiac arrest).

Discussion: Results are expected to be publicly available in 2012.

Keywords: Preterm birth, Magnesium sulphate, Individual patient data, Cerebral palsy, Meta-analysis

\section{Background}

Preterm birth and neurological outcome: The burden of disease

Babies born preterm compared with those born at term have a higher chance of dying in the first few weeks of life. Babies who survive have a greater risk of neurologic impairment, such as cerebral palsy, blindness, deafness, or cognitive dysfunction (either intellectual impairment or developmental delay), and a greater risk of substantial disability as a result of these neurologic impairments $[1,2]$. Moreover, as the rate of preterm birth in many

\footnotetext{
Correspondence: caroline.crowther@adelaide.edu.au

${ }^{1}$ Australian Research Centre for Health of Women and Babies (ARCH),

Discipline of Obstetrics and Gynaecology, Women's and Children's Hospital,
} The University of Adelaide, Adelaide, Australia countries has been rising, now up to $13 \%$ in the United States [3] and 8\% in Australia [4], more babies are at risk of death and adverse neurological outcomes. Cerebral palsy and cognitive dysfunction are the most frequent neurologic impairments, and any therapy that can reduce their prevalence would have a substantial effect on reducing overall neurologic impairments and disabilities in surviving preterm infants.

Cerebral palsy is a term which includes a number of different diseases or conditions that can arise at any time during brain development. Cerebral palsy involves a disorder of movement or posture, or both, and a disorder of motor function which is permanent but may change over time [5]. The cerebral palsies remain the most frequent cause of severe motor disability in 
childhood with a background prevalence of two per thousand live births [5]. Over $90 \%$ of affected children with cerebral palsy are expected to survive to 20 years of age [6], contributing substantially to the burden of illness into adulthood.

Very preterm birth (less than 32 weeks' gestation) and very low birthweight (less than $1500 \mathrm{~g}$ ) are principal risk factors for cerebral palsy $[7,8]$, responsible for between $17 \%$ to $32 \%$ of all cases of cerebral palsy.

Evidence from population-based registries generally shows increasing rates of cerebral palsy as gestational age decreases; with overall rates of cerebral palsy now generally considered to be stable over time [9-11] or decreasing [12]. Intraventricular haemorrhage (IVH) is a known risk factor for the later development of cerebral palsy $[13,14]$ with the rates of IVH and periventricular leukomalacia, another risk for cerebral palsy, increasing the earlier the gestational age at birth.

\section{Biological plausibility and early evidence for effect of antenatal use of magnesium sulphate on neurodevelopment}

In humans, magnesium is essential for key cellular processes, including glycolysis, oxidative phosphorylation, protein synthesis, DNA and RNA aggregation and maintenance of plasma membrane integrity [15-17]. Magnesium favourably affects mechanisms implicated in cell death by decreasing proinflammatory cytokines or free radicals produced during hypoxic-ischaemic reperfusion and inflammatory diseases of pregnancy $[15,18]$. Magnesium prevents excitotoxic calcium-induced injury [19], by a non-competitive voltage-dependent inhibition of the $\mathrm{N}$ methyl-D-aspartate receptor to glutamate which reduces calcium entry into the cell [20]. Magnesium has some beneficial haemodynamic effects including stabilising blood pressure during the first two days of life in preterm neonates [21], and may increase cerebral blood flow by reducing constriction of the cerebral arteries [22].

Transplacental transfer of magnesium is rapid with magnesium concentrations increased in fetal serum within one hour of maternal intravenous administration [23].

The first report that antenatal magnesium sulphate was associated with a reduced risk of IVH, from $18.9 \%$ to $4.4 \%$, in very low birth weight babies (less than 1500 g) came in 1992 [13] followed by a case-control analysis from the California Cerebral Palsy project investigating whether in utero exposure to magnesium sulphate was associated with a lower prevalence of cerebral palsy in low birth weight infants [24]. Cases were children with cerebral palsy who were singletons and whose birthweight had been less than 1500 g. Controls were randomly sampled from live births of less than $1500 \mathrm{~g}$ from the same birth populations. Magnesium sulphate given to the mother during labour was associated with a dramatic reduction in the risk of cerebral palsy (odds ratio $0.14 ; 95 \%$ confidence interval (CI) 0.05 to 0.51 ).

Other observational studies have supported a reduction in cerebral palsy in preterm babies after maternal administration of magnesium sulphate [25-27] and some have found a reduction in the risk of IVH $[25,28]$ and perinatal mortality [29]. However, not all observational studies have reported benefit on the risk of IVH for antenatal magnesium sulphate [30-32], cerebral palsy $[31,33,34]$, or perinatal mortality [30].

Magnesium sulphate, by its peripheral vasodilator effects when infused intravenously, produces a sensation of warmth, flushing and sweating. Reported maternal sideeffects, related to dosage and speed of infusion, include nausea, vomiting, headache and palpitations. Magnesium sulphate also acts as a neuromuscular blocking agent that causes abolition of tendon reflexes [35]. Magnesium could aggravate the cardiovascular or neuromuscular side-effects of other drugs such as betamimetics, calcium-channel blockers, digitalis and gentamicin. Infusion to concentrations above the recommended therapeutic range can lead to respiratory depression, respiratory arrest, cardiac arrest and death. For the neonate, hypermagnesaemia can lead to hyporeflexia, poor sucking, and, rarely, respiratory depression needing mechanical ventilation [36,37].

\section{Summary of the Cochrane systematic review (aggregate data)}

The Cochrane systematic review assessing the use of magnesium sulphate for women at risk of preterm birth for neuroprotection of the fetus [38] includes five trials (6145 babies): two from the US; the MagNet Trial [39] and the Beam Trial [40], one from Australia and New Zealand; the ActoMgSo4 Trial [41], one from France; the PreMag Trial [42], and one that was worldwide, but predominantly from developing countries; the Magpie Trial [43]. The first four trials specifically targeted women likely to give birth early and magnesium was used for neuroprotection, although one study, the MagNet trial [39], also had a tocolytic arm. The fifth study, the Magpie Trial was designed to prevent eclampsia in women with pre-eclampsia and included women at all gestational ages. Data from the Magpie Trial study relevant to women randomised before birth and at less than 37 weeks were provided by the authors for inclusion in the Cochrane review.

There was diversity in the inclusion and exclusion criteria for the five included trials. In particular there was a wide variation in reasons women were at risk of preterm birth (preterm labour, preterm prelabour rupture of the membranes, pre-eclampsia), the gestational age women were eligible, time of treatment prior to expected preterm birth and drug regimens (Table 1).

Paediatric mortality was reported in five trials with 6145 babies. Antenatal magnesium sulphate treatment 
Table 1 Eligible trials with their inclusion criteria and magnesium regimens as at September 2011

\begin{tabular}{|c|c|c|c|c|}
\hline Trial & $\begin{array}{l}\text { GA at } \\
\text { entry } \\
\text { (weeks) }\end{array}$ & Treatment intent & Magnesium sulphate regimen & Comparator \\
\hline \multirow[t]{2}{*}{ Mittendorf 2002 [39] } & 25 to 33 & $\begin{array}{l}\text { Tocolysis (cervical dilatation } \\
<4 \mathrm{~cm} \text { ) or; }\end{array}$ & $4 \mathrm{~g}$ bolus followed by 2 to $3 \mathrm{~g} /$ hour maintenance & $\begin{array}{l}\text { Alternative } \\
\text { tocolytic }\end{array}$ \\
\hline & & $\begin{array}{l}\text { Neuroprotection of the fetus/ } \\
\text { infant/child (dilatation } \geq 4 \mathrm{~cm} \text { ) }\end{array}$ & $4 \mathrm{~g}$ bolus (no repeats) & Placebo \\
\hline Rouse 2008 [40] & 24 to 32 & $\begin{array}{l}\text { Neuroprotection of the fetus/ } \\
\text { infant/child }\end{array}$ & $\begin{array}{l}6 \mathrm{~g} \text { over } 20-30 \text { mins followed by } 2 \mathrm{~g} / \mathrm{hr} \text { (retreatment } \\
\text { permitted) }\end{array}$ & Placebo \\
\hline Crowther 2003 [41] & $\begin{array}{l}\text { less than } \\
30\end{array}$ & $\begin{array}{l}\text { Neuroprotection of the fetus/ } \\
\text { infant/child }\end{array}$ & $\begin{array}{l}4 \mathrm{~g} \text { over } 20 \text { mins, then } 1 \mathrm{~g} / \text { hour for up to } 24 \text { hours or } \\
\text { birth (no repeats) }\end{array}$ & Placebo \\
\hline Marret 2008 [42] & $\begin{array}{l}\text { less than } \\
33\end{array}$ & $\begin{array}{l}\text { Neuroprotection of the fetus/ } \\
\text { infant/child }\end{array}$ & $4 \mathrm{~g}$ (no repeats) & Placebo \\
\hline $\begin{array}{l}\text { The Magpie Trial } \\
\text { Collaborative Group } 2002 \\
\text { [43] }\end{array}$ & $\begin{array}{l}\text { less than } \\
37\end{array}$ & $\begin{array}{l}\text { Neuroprotection of the } \\
\text { pre-eclamptic mother }\end{array}$ & $\begin{array}{l}4 \mathrm{~g} \text { over } 10-15 \text { mins, then either } 1 \mathrm{~g} / \text { hour for } 24 \text { hours, } \\
\text { or } 5 \mathrm{~g} \text { every } 4 \text { hours IM for } 24 \text { hours (no repeats) }\end{array}$ & Placebo \\
\hline
\end{tabular}

had no overall significant effect on total paediatric mortality overall (risk ratio (RR) $1.04,95 \%$ CI 0.82 to 1.17 , five trials, 6145 infants). While results between treatment intent did not differ, there was substantial heterogeneity $\left(\mathrm{I}^{2}=45 \%\right)$ between studies for this outcome, largely due to the other intent (tocolytic) arm of the MagNet study (RR 15.8, 95\% CI 0.93 to 267, 106 infants).

Cerebral palsy was reported in five trials with 6145 infants. There was a $32 \%$ relative reduction in risk of cerebral palsy with antenatal magnesium sulphate treatment, (overall RR $0.68,95 \%$ CI 0.54 to 0.87 ). This remained significant within the neuroprotective intent group (RR 0.71, 95\% CI 0.55 to 0.91 , four trials, 4446 infants). Overall, the number needed to treat to prevent one case of cerebral palsy was 63 (95\% CI 39 to 172).

Antenatal magnesium sulphate treatment had no overall significant effect on the combined outcome of paediatric mortality or cerebral palsy, but there was substantial heterogeneity overall $\left(\mathrm{I}^{2}=51.3 \%\right)$. However, there was a significant reduction in the combined outcome of paediatric mortality or cerebral palsy in the neuroprotection subgroup (RR $0.85,95 \%$ CI 0.74 to 0.98 , four trials, 4446 infants), with little evidence of heterogeneity $\left(\mathrm{I}^{2}=5.3 \%\right)$. The number needed to treat for an extra survivor free of cerebral palsy in the neuroprotective subgroup was 41 (95\% CI 22 to 357).

\section{Need for an individual participant data meta-analysis}

Aggregate data analysis methods such as those used in the Cochrane review are limited because, for example, results for women with different levels of risk have been averaged, potentially concealing any different effects by reasons that women were considered to be at risk for preterm birth. Similarly wide within-trial variation in different degrees of effect of magnesium sulphate by gestational age is concealed by aggregation. Aggregated outcomes such as 'the number of children developing cerebral palsy' include a range of severity of the disorder. The published aggregate data is variable in completeness of reporting outcomes and in the definitions used within the trials. Not all trials have published the same combined outcomes and very few subgroup analyses using similar subgroup categories (such as gestational age) have been published, making aggregation of these results impossible.

Many questions remain. Is antenatal magnesium sulphate treatment more effective in some women by reason of their risk of preterm birth? What is the gestational age range for maximal benefit? What dose and timing prior to birth of magnesium is best? Is maintenance treatment or re-treatment necessary?

The best way to address the limitations of the aggregate meta analysis is to use the large amount of existing trial data and conduct an individual participant data (IPD) review and meta-analysis, rather than undertake another trial which would need to be much larger than any of the existing trials to be able to address the multiple subgroup analyses with sufficient power.

Analysis of thoroughly checked and updated data from individual participants in all the available randomised trials has been described as the gold standard in systematic reviews [44]. Estimates of treatment effects are often different from those obtained from aggregate published data due to the ability to include additional unpublished data and to categorise all participants according to standardised definitions. The methods and advantages of IPD review have been well described $[45,46]$.

Advantages that address the limitations of aggregate data analyses reported include the ability to:

- undertake subgroup analyses to examine important hypotheses about differences in treatment effect that cannot be achieved with the published aggregate data, 
- explore interactions between treatment and participant-level characteristics,

- collect uniformly defined, relevant data for all trials.

An integral component of conducting this IPD metaanalysis is the formation of an international collaborative group of trialists where all researchers endorse the IPD protocol and provide data from their trials. This generates additional benefits that include:

- more complete identification of trials and of trial details,

- compliance with standard definitions, provision of missing data on characteristics of trials, all women who were randomised and their babies, and outcomes,

- more balanced interpretation, endorsement and global dissemination of results, and

- better clarification and consensus on future research needed with the opportunity for ongoing international collaborations.

\section{Objectives}

To assess, using IPD meta-analyses, the effects of administration of antenatal magnesium sulphate given to women at risk of preterm birth on important clinical outcomes, and whether treatment effects differ depending on important prespecified participant and treatment characteristics:

- the reason the woman was considered to be at risk of preterm birth (such as preterm labour, hypertensive disease of pregnancy, antepartum haemorrhage, presence or absence of ruptured membranes at trial entry),

- the primary reason antenatal magnesium sulphate treatment was given (such as neuroprotection of the fetus, pre-eclampsia, or tocolysis),

- the number of babies in utero (singleton or multiple),

- the gestational age when antenatal magnesium sulphate treatment was given,

- the time prior to birth antenatal magnesium sulphate treatment was given,

- the type, mode of administration and dosage of antenatal magnesium sulphate planned and given,

- whether maintenance treatment with antenatal magnesium sulphate was used, and

- whether repeat antenatal magnesium sulphate treatment was used.

\section{Methods/design \\ Study design}

IPD meta-analysis.

\section{Criteria for the studies for inclusion and exclusion}

Studies, published or unpublished, will be considered eligible if they randomised women considered at risk of preterm birth (less than 37 weeks' gestation) to either magnesium sulphate or a suitable control treatment with adequate allocation concealment. Trials will be included if the primary aim of the study was to prevent neurologic abnormalities in the unborn baby, or if the primary aim was otherwise but long-term neurological outcomes were reported for the infants. Quasi-random study designs will be excluded. See Table 1 for a summary of the eligible trials as at September 2011.

Eligibility of trials will be assessed independently and unblinded for author and journal by two members of the AMICABLE IPD Project Team. Any differences in opinion regarding eligibility will be resolved by discussion. If IPD are unavailable from any eligible trial, the trial will be included in the review and aggregated and stratified data will be used for sensitivity analyses were possible.

\section{Search strategy to identify potential trials}

We will use the search strategy developed by the Cochrane Pregnancy and Childbirth Review Group for the Cochrane review, which identifies trials from:

1. quarterly searches of the Cochrane Central Register of Controlled Trials (CENTRAL);

2. weekly searches of MEDLINE;

3. handsearches of 30 journals and the proceedings of major conferences;

4. weekly current awareness alerts for a further 44 journals plus monthly BioMed Central email alerts.

We will also search CENTRAL, MEDLINE and EMBASE to identify trials published since the search cutoff date of 31 August 2008 for the Cochrane review; using the terms [antenatal or prenatal] and [magnesium] and [preterm or premature or neuroprotection or 'cerebral palsy'].

In addition we will access Current Controlled Trials http://www.controlled-trials.com and the Australian and New Zealand Trials Register http://www.anzctr.org.au to identify recently completed or ongoing trials.

Experts in the field and trialists will be asked if they know of any unpublished or other trials.

\section{Data collection and management}

A new set of pre-specified and clearly defined variables (both for participant-level and trial-level factors as well as for outcomes) and a newly developed coding system will be used. Data will be collected on all women randomised coded for anonymity, (date of birth, centre identification); baseline data for descriptive purposes and analyses (reason at risk of preterm birth, gestational age at trial entry, plurality of the pregnancy, expected date of delivery); details of the intervention given (date of randomisation, allocated intervention, type and dose of magnesium sulphate given, mode of administration, whether maintenance dose given, whether re-treatment 
given and amount); and maternal and infant outcomes listed below to allow planned analyses.

Trialists will provide de-identified IPD in any convenient format by encrypted, electronic transfer where possible or other means as needed. The individual trial data will be recoded as required and stored in a custom designed secure database which will be only accessible by authorised personnel of the AMICABLE Data Management Group. Trialists will be asked to verify all recoded data prior to any analysis and the data will not be used for any other purpose without permission of all collaborators.

Data will be checked with respect to range, internal consistency, missing or extreme values, errors and consistency with published reports. Trial details such as randomisation methods and intervention details will be cross-checked against published reports, trial protocols and data collection sheets. Inconsistencies or missing data will be discussed with the individual trialists and attempts will be made to resolve any problems by consensus. Each trial will be analysed individually, and the resulting analyses and trial data will be sent to the trialists for verification.

We will assess risk of bias for each study using the criteria outlined in the Cochrane Handbook for Systematic Reviews of Interventions [47]. These criteria are:

1. Random sequence generation (checking for possible selection bias)

2. Allocation concealment (checking for possible selection bias)

3. i. Blinding of participants and personnel (checking for possible performance bias)

ii. Blinding of outcome assessment (checking for possible detection bias)

4. Incomplete outcome data (checking for possible attrition bias due to the amount, nature and handling of incomplete outcome data)

5. Selective reporting (checking for reporting bias)

6. Other bias (checking for bias due to problems not covered by 1 to 5 above)

We will make explicit judgements about whether studies are at high, low or unclear risk of bias, according to the criteria given in the Handbook; and we will assess the likely magnitude and direction of the bias and whether we consider it is likely to impact on the findings.

\section{Data items to be collected \\ Trial level information}

1. Dates the trial opened and closed accrual

2. Number of women randomised

3. Informed consent procedures
4. Methods of random allocation

5. Stratification factors used

6. Methods of allocation concealment

7. Blinding of outcome assessment

8. Purpose magnesium sulphate treatment given (neuroprotection for the fetus, neuroprotection for the mother, tocolysis, pre-eclampsia, other)

9. Details of the planned intervention in the experimental arm

10. Details of the planned intervention in the control arm

\section{Participant-level information: maternal characteristics at} trial entry

1. Unique identification coded for anonymity

2. Maternal age

3. Parity

4. Ethnicity

5. Public or private patient

6. Previous obstetric history

7. Reason the woman was considered to be at risk of preterm birth (such as preterm labour/pre-eclampsia/ placenta abruption/placenta previa/chorioamnionitis/ other antepartum haemorrhage/preterm rupture of membranes)

8. Number of babies in-utero (singleton, twin or higher order multiple pregnancy)

9. Gestational age at trial entry

Participant-level information: maternal outcomes after trial entry

1. Actual treatment dose details (loading dose given, maintenance dose, retreatment dose)

2. Time from antenatal magnesium sulphate treatment to birth

3. Adverse events for the woman at time of treatment

4. Intrapartum information

5. Postnatal information

\section{Participant-level information: infant outcomes}

1. Unique baby identification and mother identification coded for anonymity

2. Date and time of birth

3. Gestational age at birth

4. Gender

5. Mode of birth

6. Birth weight, length, head circumference

7. Apgar score at 1 and 5 minutes

8. Neonatal complications/status

9. Mortality and age at death

10. Cause of death

11. Childhood follow-up assessments

\section{Planned analyses}

A detailed statistical analysis plan will be prepared by the AMICABLE Data Management Group and agreed upon by the AMICABLE IPD Project Team and the 
AMICABLE Group prior to the data analyses. Any analyses conducted will be based on the checked and updated IPD from all available trials. All randomised participants with outcome data available will be included in the analyses, which will be performed on an intention to treat basis, according to the treatment allocation at randomisation.

For each of the outcomes a one stage approach to analysis will be taken so that the IPD from all eligible trials are included in a single model. Fitting a single model for each outcome variable will enable the variation across trials to be accounted for within the model. A treatment by trial interaction term will be tested to assess heterogeneity of treatment effect across trials. If excessive statistical heterogeneity in treatment effect or inconsistency across trials is detected (i.e. if the trial by treatment interaction term is significant), then the rationale for combining trials will be questioned and the source of heterogeneity explored.

Binary outcomes will be analysed using log binomial regression models and results will be presented as RR with 95\% CI and associated two-sided $\mathrm{p}$ values. Continuous outcomes will be analysed using linear regression models and results will be presented as differences in means with 95\% CI and two-sided p values. Correlation between outcomes due to multiple births will be taken into account using generalized estimating equations as appropriate.

Any differences in treatment effect between prespecified subgroups will be assessed by testing a treatment by subgroup interaction term within the model.

Where data are missing, those patients will be removed from the analysis. Reasons for missingness will be explored where possible. For each outcome if there are unbalanced or large amounts of missing endpoint data for at least one trial then sensitivity analyses will be undertaken to assess the impact of removing such trials from the analysis.

\section{Outcomes}

Outcomes have been chosen to be most representative of the clinically important measures of effectiveness and safety, including serious outcomes, for women and their babies.

\section{Primary Outcomes}

For the infants/children

-death or cerebral palsy

For the women

-any severe maternal outcome potentially related to treatment (death, respiratory arrest or cardiac arrest)

\section{Secondary Outcomes}

For the infants

-gestational age at birth

-birth weight (raw values and Z scores) [48]

-head circumference at birth (raw values and $\mathrm{Z}$ scores) [48]

-length at birth (raw values and $\mathrm{Z}$ scores) [48]

-Apgar score less than 7 at five minutes

- use of active resuscitation at birth

-use of ongoing respiratory support

-chronic lung disease/bronchopulmonary dysplasia

(as defined by trialists)

-neonatal convulsions

-neonatal encephalopathy

•any IVH

-severe IVH (grade 3 or 4)

-cystic periventricular leukomalacia

- posthaemorrhagic hydrocephaly or ventriculomegaly

-proven systemic neonatal infection

-necrotising enterocolitis

-patent ductus arteriosus requiring treatment

-retinopathy of prematurity

\section{For the children (follow up)}

-death (fetal, neonatal or later death up to the time of follow up) and cause of death

-cerebral palsy (any cerebral palsy, as defined by the trialists)

-severity of cerebral palsy (categorised as, mild, moderate or severe, as defined by the trialists)

-severe adverse neonatal outcome (defined as death, chronic lung disease, patent ductus arteriosus requiring treatment, neonatal encephalopathy, necrotising enterocolitis, stage 3 or worse retinopathy of prematurity, grade 3 or $4 \mathrm{IVH}$ )

-other neurosensory impairments (other than cerebral palsy)

odevelopmental delay or intellectual impairment (categorised as nil, mild, moderate or severe, by the trialists)

- blindness (defined as visual acuity worse than $6 / 60(20 / 200)$ in the better eye)

odeafness (defined as hearing loss requiring amplification or worse)

ogross motor dysfunction (defined as mild, moderate or severe, by trialists or by the Gross Motor Classification System [score 1-5], if available) [49]

opsychomotor dysfunction (categorised as nil, mild (less than 85), moderate (less than 70) or severe (less than 55) by the Psychomotor 
Developmental Index (PDI)) on the Bayley Scales of Infant Development [50]

-any neurosensory disability (defined as developmental delay or intellectual impairment [developmental quotient or intelligence quotient more than one SD below the mean], cerebral palsy [abnormality of muscle tone with motor dysfunction], blindness, or deafness, at follow up later in childhood)

-major neurosensory disability (defined as any moderate or severe neurosensory impairment)

- death or substantial gross motor dysfunction (defined as death, or motor dysfunction (such that the child was not walking at age two years or later, or the inability to grasp and release a small block with both hands at two years or later))

-death or neurosensory disability (defined as death or any of the neurosensory impairments)

-death or major neurosensory disability (defined as death or any moderate or severe neurosensory impairment)

-growth assessments at childhood follow up for:

Oweight (raw values and $\mathrm{Z}$ scores) [51]

ohead circumference (raw values and $\mathrm{Z}$ scores) [51]

oheight (raw values and $\mathrm{Z}$ scores) [51]

-other developmental assessments (as used by the trialists)

\section{For the women}

-adverse effects severe enough to stop treatment -postpartum haemorrhage

-mode of birth

-chorioamnionitis during labour

-intrapartum fever requiring the use of antibiotics

-length of postnatal stay

•individual components of maternal primary outcome

\section{Planned subgroup analyses}

Where data exist, subgroup comparisons will be conducted for the following outcomes;

-death or cerebral palsy

-death

-cerebral palsy

-severe maternal adverse event potentially related to treatment

-death or major neurosensory disability

Any differences in treatment effect between subgroups will be assessed by testing a treatment by subgroup interaction term within the model.

\section{Subgroups}

1) primary reason pregnancy was considered to be at high risk of preterm delivery (preterm labour/preeclampsia/placenta abruption/placenta previa/chorioamnionitis/other antepartum haemorrhage/prelabour rupture of the membranes)

2) purpose of treatment - neuroprotection of the fetus/neuroprotection of the mother/pre-eclampsia/ eclampsia/tocolysis/other)

3) multiple birth

4) gestational age when antenatal magnesium sulphate treatment was given

a) categories: $<26 ; 26$ to $<28 ; 28$ to $<30 ; 30$ to $<32$; 32 to $<34$ completed weeks at randomisation; (groups will be combined if data are insufficient);

b) categories: < 34; $\geq 34$ completed weeks at randomisation;

c) categories: < 32; $\geq 32$ completed weeks at randomisation;

d) categories: < 30; $\geq 30$ completed weeks at randomisation;

e) categories: $<28$; $\geq 28$ completed weeks at randomisation.

5) time from antenatal magnesium sulphate treatment to birth ( 0 to $<4$ hours; 4 to $<8$ hours; 8 to $<$ 12 hours; 12 to $<24$ hours (groups will be combined if data are insufficient)

6) mode of administration (intravenous, intramuscular)

7) actual dose of antenatal magnesium sulphate received

a) actual total trial dose received ( $<4 \mathrm{~g} ; 4$ to $<$

$14 \mathrm{~g} ; 14$ to $<28 \mathrm{~g} ; \geq 28 \mathrm{~g}$ );

b) actual total trial dose received adjusted for pre-trial dose received $(<4 \mathrm{~g} ; 4$ to $<6 \mathrm{~g} ; 6$ to $<$ $14 \mathrm{~g} ; 14$ to $<28 \mathrm{~g} ; \geq 28 \mathrm{~g}$ );

c) pre-trial dose received or not

8) whether maintenance treatment with antenatal magnesium sulphate was used

9) whether repeat antenatal magnesium sulphate treatment was used

\section{Planned sensitivity analyses}

To assess whether the results are robust to trial design, sensitivity analyses will be performed by excluding trials with high rates of participant exclusions, where losses are considered to have the potential to impact on the results.

To assess whether the results are robust to the inclusion or exclusion of trials where IPD are unavailable 
sensitivity analyses will be undertaken where IPD will be combined with available aggregate data from such trials.

\section{Multiple comparisons}

A large number of outcomes are being investigated in this study which increases the chance of observing 'false positive' results. However, all outcomes are important in giving a full clinical picture that considers the benefits and risks to both mothers and infants. We do not plan formal statistical adjustment of $\mathrm{p}$ values planned to account for multiple comparisons due to the non-independence of outcomes in this study. Results will be interpreted with caution.

\section{Ethics and management issues Ethical considerations}

Participants in the individual trials have previously given informed consent to participate in their respective trial. The data for this project are to be used for the purpose for which they were originally collected and are available through an agreement between all trialists of the collaborative group. These trialists remain custodians of their original individual trial data at all times.

\section{Project management}

For the purpose of this project, an international Collaborative Group, The AMICABLE Group, was formed and consists of the following working groups with specific responsibilities and tasks:

\section{The AMICABLE IPD Project Team}

The AMICABLE IPD Project Team is the Steering Group which is responsible for the project's management decisions and the daily management of the Collaboration. The Project Team's tasks are to design the project's protocol and analysis plan, organise The AMICABLE Group Meetings and act as a liaison between all the members of The AMICABLE Group. The AMICABLE IPD Project Team will meet regularly every two to four months, usually by teleconference.

\section{The AMICABLE Trialist Group}

The AMICABLE Group will consist normally of two members from each eligible trial. The first author for each trial, will be invited to become a member of the Collaboration. In order to keep The AMICABLE Group updated, authors of new trials or previously unknown trials may be contacted and invited to join the Collaboration in the course of the project.

\section{AMICABLE Data Management Group}

The data management group will be convened by the Chair of The AMICABLE IPD Project Team and comprises the statisticians from the data management centre and participating trials who will conduct the analyses. The data management group will be responsible for the storage and analyses of the IPD project data.

\section{AMICABLE Group Meetings}

The AMICABLE Group face-to-face meetings will be organised at least twice during the study. Representatives of all eligible trials will be invited to attend the meetings. The meetings will be scheduled, if possible, in conjunction with international conferences. During those meetings, various aspects of the project will be discussed with all the collaborators, such the project's design and conduct, the analysis plan, and the interpretation and reporting of the results. The final collaborators' meeting is scheduled for late 2012.

\section{Publication of results}

The final results of the study will be presented to the collaborators for discussion. The main manuscript will be prepared by The AMICABLE IPD Project Team and circulated to The AMICABLE Group and The AMICABLE Data Management Group for comment and revision. The revised draft paper will be circulated for final comment and agreement prior to publication. Publications will be authored in the name of The AMICABLE Group. The names of all participating collaborators will be acknowledged within the manuscript.

\section{Discussion}

Babies born preterm compared with those born at term have a higher chance of dying in the first few weeks of life. Babies who survive have a high risk of substantial disability from neurosensory impairments such as cerebral palsy, blindness, deafness, or cognitive dysfunction.

The Cochrane review on antenatal magnesium sulphate given to women at risk of preterm birth has identified an important reduction in the risk of cerebral palsy. However it remains unclear what the role of antenatal magnesium sulphate is in clinical practice.

This IPD meta-analysis, using data already collected in the individual trials may reveal which women and/or babies might benefit more from antenatal magnesium sulphate. This approach has been described as the 'gold standard' of systematic review methodology as it allows for more powerful and flexible analysis of both subgroups and outcomes. The international collaboration of trialists will guarantee exceptional data availability and quality, and ensure endorsement and global implementation of recommendations into clinical practice and policy.

The AMICABLE Group was formed in 2009 to undertake a meta-analysis based on IPD. Provision of data by 
the participating collaborators commenced in 2010 and results will be ready for presentation in 2012 .

\author{
Abbreviations \\ AMICABLE: Antenatal magnesium individual participant data (IPD) \\ international collaboration: assessing the benefits for babies using the best \\ level of evidence; CENTRAL: Cochrane central register of controlled trials; Cl: \\ Confidence interval; IVH: Intraventricular haemorrhage; IPD: Individual \\ participant data; RR: Risk ratio.
}

\section{Acknowledgements}

The AMICABLE Group

The AMICABLE IPD Project Team

CA Crowther (chair), PF Middleton' (epidemiologist), LM Askie ${ }^{2}$ (advice on IPD methodology), LW Doyle (advice on paediatric aspects), TK Bubner ${ }^{1}$ (project coordinator), M Voysey ${ }^{4}$ (AMICABLE coordinating statistician). 'Australian Research Centre for Health of Women and Babies, Discipline of Obstetrics and Gynaecology, The University of Adelaide, Australia ${ }^{2}$ NHMRC Clinical Trials Centre, University of Sydney, Australia

${ }^{3}$ Department of Obstetrics and Gynaecology, University of Melbourne, Australia

${ }^{4}$ Centre for Statistics in Medicine, University of Oxford, United Kingdom The AMICABLE Data Management Group (as at 11/8/11):

M Voysey (coordinating statistician), L Yelland (participating trial statistician), $S$ Zhang (data management centre).

The AMICABLE Trialist Group (as at 11/8/2011):

Australasian Collaborative Trial of Magnesium Sulphate (ACTOMgSO4): CA Crowther, LW Doyle;

MAGnesium sulphate for Prevention of Eclampsia (MAGPIE): LW Duley, D Altman;

Magnesium sulfate given before very pre-term birth (PREMAG): S Marret, L Marpeau, C Leveque;

Magnesium and Neurologic Endpoints Trial (MAGNET): R Mittendorf, P Pryde. The AMICABLE IPD Study is funded by a project grant from the Australian National Health and Medical Research Council (NHMRC) which will support the collection of the IPD by the original investigators, data management and the analyses and the collaborators' meetings. The NHMRC is not involved in any other aspect of the project, such as the design of the project's protocol and analysis plan, the collection and the analyses of the project's data, or the interpretation and the publication of the study results.

\section{Authors' contributions}

The Chair of The AMICABLE Project Team (CAC) wrote the first draft of the IPD protocol and prepared the initial draft of the manuscript. The AMICABLE Project Team and The AMICABLE Data Management Group participated in the protocol development and commented on all drafts of the manuscript. The AMICABLE Group participated in the development of the IPD protocol and have read and approved the final draft of the manuscript.

\section{Competing interests}

The authors declare that they have no competing interests.

Received: 5 December 2011 Accepted: 21 March 2012 Published: 21 March 2012

\section{References}

1. Doyle LW, Victorian Infant Collaborative S: Outcome at 5 years of age of children 23 to 27 weeks' gestation: Refining the prognosis. Pediatrics 2001, 108(1):134-141.

2. Saigal S, Doyle LW: An overview of mortality and sequelae of preterm birth from infancy to adulthood. Lancet 2008, 371(9608):261-269.

3. Martin JA, Hamilton BE, Sutton PD, Ventura SJ, Menacker F, Kirmeyer S, Munson ML: Births: final data for 2005. Natl Vital Stat Rep 2007, 56(6):1-103.

4. Laws P, Li Z, Sullivan EA: Australia's mothers and babies 2008, Perinatal statistics series no. 24. Cat. no. PER 50 Canberra: AlHW; 2010.

5. Oxford Register of Early Childhood Impairment: National Perinatal Epidemiology Unit 2001 Annual Report Oxford: Institue of Health Sciences; 2001.

6. Hutton JL, Cooke T, Pharoah POD: Life expectancy in children with cerebral palsy. Brit Med J 1994, 309(6952):431-435.
7. Winter S, Autry A, Boyle C, Yeargin-Allsopp M: Trends in the prevalence of cerebral palsy in a population-based study. Pediatrics 2002, 110(6):1220-1225.

8. Drummond PM, Colver AF: Analysis by gestational age of cerebral palsy in singleton births in north-east England 1970-94. Paediatr Perinat Epidemiol 2002, 16(2):172-180.

9. Himpens E, Van den Broeck C, Oostra A, Calders P, Vanhaesebrouck P. Prevalence, type, distribution, and severity of cerebral palsy in relation to gestational age: a meta-analytic review. Dev Med Child Neurol 2008, 50(5):334-340.

10. Glinianaia S, Rankin J, Selvin S, Nelson KB: Cerebral palsy rates by birth weight, gestation and severity in North of England, 1991-2000 singleton births. Arch Dis Child 2011, 96:180-185.

11. Stanley FJ, Watson L: Trends in Perinatal-Mortality and Cerebral-Palsy in Western-Australia, 1967 to 1985. Brit Med J 1992, 304(6843):1658-1663.

12. Sellier E, Surman G, Himmelmann K, Andersen G, Colver A, Krageloh-Mann I, De-la-Cruz J, Cans C: Trends in prevalence of cerebral palsy in children born with a birthweight of 2,500 g or over in Europe from 1980 to 1998. Eur J Epidemiol 2010, 25(9):635-642.

13. Kuban KC, Leviton A, Pagano M, Fenton T, Strassfeld R, Wolff M: Maternal toxemia is associated with reduced incidence of germinal matrix hemorrhage in premature babies. J Child Neurol 1992, 7(1):70-76.

14. Ancel PY, Livinec F, Larroque B, Marret S, Arnaud C, Pierrat V, Dehan M, N'Guyen S, Escande B, Burguet A, et al: Cerebral palsy among very preterm children in relation to gestational age and neonatal ultrasound abnormalities: the EPIPAGE cohort study. Pediatrics 2006, 117(3):828-835.

15. Hoffman DJ, Marro PJ, McGowan JE, Mishra OP, Delivoriapapadopoulos M: Protective effect of $\mathrm{MgSO} 4$ infusion on nmda receptor binding characteristics during cerebral cortical hypoxia in the newborn piglet. Brain Res 1994, 644(1):144-149.

16. Mildvan AS: Role of magnesium and other divalent cations in ATPutilizing enzymes. Magnesium 1987, 6(1):28-33.

17. McIntosh TK, Vink R, Yamakami I, Faden Al: Magnesium protects against neurological deficit after brain injury. Brain Res 1989, 482(2):252-260.

18. Shogi T, Miyamoto A, Ishiguro S, Nishio A: Enhanced release of IL-1 beta and TNF-alpha following endotoxin challenge from rat alveolar macrophages cultured in low-Mg2+ medium. Magnesium Res 2003, 16(2):111-119.

19. Nowak L, Bregestovski P, Ascher P, Herbet A, Prochiantz A: Magnesium gates glutamate-activated channels in mouse central neurons. Nature 1984, 307(5950):462-465.

20. Ovbiagele B, Kidwell CS, Starkman S, Saver JL: Neuroprotective agents for the treatment of acute ischemic stroke. Curr Neurol Neurosci Rep 2003, 3(1):9-20.

21. Rantonen TH, Gronlund JU, Jalonen JO, Ekblad UU, Kaapa PO, Kero PO, Valimaki IAT: Comparison of the effects of antenatal magnesium sulphate and ritodrine exposure on circulatory adaptation in preterm infants. Clin Physiol Funct Imaging 2002, 22(1):13-17.

22. Macdonald RL, Curry DJ, Aihara Y, Zhang ZD, Jahromi BS, Yassari R: Magnesium and experimental vasospasm. J Neurosurg 2004, 100(1):106-110.

23. Hallak M, Cotton DB: Transfer of maternally administered magnesium sulfate into the fetal compartment of the rat. Am J Obstet Gynecol 1993, 169(2):427-431.

24. Nelson KB, Grether JK: Can magnesium sulphate reduce the risk of cerebral palsy in very low birthweight infants? Pediatrics 1995, 95:1-1.

25. Wiswell TE, Graziani LJ, Caddell JL, Vecchione N, Stanley C, Spitzer AR: Maternally-administered magnesium sulfate (MgSO4) protects against early brain injury and long-term adverse neurodevelopmental outcomes in preterm infants: a prospective study. Pediatr Res 1996, 39(4):1502-1502.

26. Hauth JC, Goldenberg RL, Nelson KG, Dubard MB, Peralta MA, Gaudier FL: Reduction of cerebral palsy with maternal MgSO4 treatment in newborns weighing 500-1000 g. Am Obstetrics \& Gynaecology 1995, 172(1 Pt 2):419.

27. Schendel DE, Berg CJ, YearginAllsopp M, Boyle CA, Decoufle P: Prenatal magnesium sulfate exposure and the risk for cerebral palsy or mental retardation among very low-birth-weight children aged 3 to 5 years. Jama-J Am Med Assoc 1996, 276(22):1805-1810.

28. FineSmith RB, Roche $K$, Yellin PB, Walsh KK, Shen C, Zeglis M, Kahn A, Fish I: Effect of magnesium sulfate on the development of cystic periventricular leukomalacia in preterm infants. Am J Perinat 1997, 14(5):303-307. 
29. Grether JK, Hoogstrate J, Selvin S, Nelson KB: Magnesium sulfate tocolysis and risk of neonatal death. Am J Obstet Gynecol 1998, 178(1):1-6.

30. Kimberlin DF, Hauth JC, Goldenberg RL, Bottoms SF, lams JD, Mercer B, MacPherson C, Thurnau GR: The effect of maternal magnesium sulfate treatment on neonatal morbidity in $<=1000$-gram infants. Am J Perinat 1998, 15(11):635-641.

31. Paneth N, Jetton J, PintoMartin J, Susser M, Clark C, Gardiner J, Holzman C, Lorenz JM, Reuss ML: Magnesium sulfate in labor and risk of neonatal brain lesions and cerebral palsy in low birth weight infants. Pediatrics 1997, 99(5):E1.

32. Weintraub Z, Solovechick M, Reichman B, Rotschild A, Waisman D, Davkin O, Lusky A, Bental Y: Israel Neonatal Network: Effect of maternal tocolysis on the incidence of severe periventricular/intraventricular haemorrhage in very low birthweight infants. Arch Dis Child 2001, 85(1): F13-F17.

33. Grether JK, Hoogstrate J, Walsh-Greene E, Nelson KB: Magnesium sulfate for tocolysis and risk of spastic cerebral palsy in premature children born to women without preeclampsia. Am J Obstet Gynecol 2000, 183(3):717-725.

34. O'Shea TM, Klinepeter KL, Meis PJ, Dillard RG: Intrauterine infection and the risk of cerebral palsy in very low-birthweight infants. Paediatr Perinat Epidemiol 1998, 12:(1):72-83.

35. Baraka A, Yazigi A: Neuromuscular interaction of magnesium with succinylcholine-vecuronium sequence in the eclamptic parturient. Anesthesiology 1987, 67(5):806-808.

36. Levene M, Blennow M, Whitelaw A, Hanko E, Fellman V, Hartley R: Acute effects of two different doses of magnesium sulphate in infants with birth asphyxia. Arch Dis Child-Fetal 1995, 73(3):F174-F177.

37. Lipsitz PJ: The clinical effects of excess magnesium in the newborn. Pediatrics 1971, 47(3):501-509.

38. Doyle LW, Crowther CA, Middleton P, Marret S, Rouse D: Magnesium sulphate for women at risk of preterm birth for neuroprotection of the fetus. Cochrane Database Syst Rev 2009, , 1: CD004661, DOl: 10.1002/ 14651858.CD004661.pub3.

39. Mittendorf R, Dambrosia J, Pryde PG, Lee KS, Gianopoulos JG, Besinger RE, Tomich PG: Association between the use of antenatal magnesium sulfate in preterm labor and adverse health outcomes in infants. Am J Obstet Gynecol 2002, 186(6):1111-1118.

40. Rouse DJ, Hirtz DG, Thom EA, et al: Magnesium sulfate for the prevention of cerebral palsy. New Engl J Med 2008, 359:895-905.

41. Crowther CA, Hiller JE, Doyle LW, Haslam RR, the Australasian Collaborative Trial of Magnesium Sulphate (ACTOMgSO4) Collaborative Group: Effect of magnesium sulfate given for neuroprotection before preterm birth-a randomized controlled trial. Jama-J Am Med Assoc 2003, 290(20):2669-2676

42. Marret $S$, Marpeau L, Benichou J: Benefit of magnesium sulfate given before very preterm birth to protect infant brain. Pediatrics 2008, 121(1):225-226.

43. Duley L, Farrell B, Armstrong N, Spark P, Roberts B, Smyth R, Tivnan M, Laws A, Corfield N, Salter A, et al: The Magpie trial: a randomised trial comparing magnesium sulphate with placebo for pre-eclampsia. outcome for children at 18 months. Bjog-Int J Obstet Gy 2007, 114(3):289-299.

44. Chalmers I: The cochrane-collaboration - preparing, maintaining, and disseminating systematic reviews of the effects of health-care. Ann Ny Acad Sci 1993, 703:156-165.

45. Stewart LA, Tierney JF: To IPD or not to IPD? Advantages and disadvantages of systematic reviews using individual patient data. Eval Health Prof 2002, 25(1):76-97.

46. Cochrane Collaboration IPD Group: Chapter 19 Reviews of individual patient data. In Cochrane Handbook for Systematic Reviews of Interventions Version 500 [updated February 2008]. Edited by: Higgins JPT, Green S. USA: John Wiley 2008:

47. Cochrane Handbook for Systematic Reviews of Interventions Version 5.1.0. In The Cochrane Collaboration Edited by: Higgins JPT, Green S 2011 [http://www.cochrane-handbook.org].

48. Roberts CL, Lancaster PAL: Australian national birthweight percentiles by gestational age. Med J Australia 1999, 170(3):114-118.

49. Palisano R, Rosenbaum P, Walter S, Russell D, Wood E, Galuppi B: Development and reliability of a system to classify gross motor function in children with cerebral palsy. Dev Med Child Neurol 1997, 39(4):214-223.
50. Bayley N: Bayley scales of infant development. 2 edition. San Antonio: Psychological Corportation; 1993.

51. WHO Multicentre Growth Reference Study Group: WHO Child Growth Standards based on length/height, weight and age. Acta Paediatr 2006, 450:76-85.

doi:10.1186/2046-4053-1-21

Cite this article as: : Antenatal magnesium individual participant data international collaboration: assessing the benefits for babies using the best level of evidence (AMICABLE). Systematic Reviews 2012 1:21.

\section{Submit your next manuscript to BioMed Central and take full advantage of:}

- Convenient online submission

- Thorough peer review

- No space constraints or color figure charges

- Immediate publication on acceptance

- Inclusion in PubMed, CAS, Scopus and Google Scholar

- Research which is freely available for redistribution

Submit your manuscript at www.biomedcentral.com/submit
Biomed Central 